University of Wollongong

Research Online

Faculty of Informatics - Papers (Archive)

Faculty of Engineering and Information

Sciences

September 2004

\title{
Power quality waveform generator based on the CSound software sound synthesizer
}

J. P. Braun

University of Wollongong

V. J. Gosbell

University of Wollongong, vgosbell@uow.edu.au

S. Perera

University of Wollongong, sarath@uow.edu.au

Follow this and additional works at: https://ro.uow.edu.au/infopapers

Part of the Physical Sciences and Mathematics Commons

\section{Recommended Citation}

Braun, J. P.; Gosbell, V. J.; and Perera, S.: Power quality waveform generator based on the CSound software sound synthesizer 2004.

https://ro.uow.edu.au/infopapers/44

Research Online is the open access institutional repository for the University of Wollongong. For further information contact the UOW Library: research-pubs@uow.edu.au 


\title{
Power quality waveform generator based on the CSound software sound synthesizer
}

\author{
Abstract \\ Power quality investigations in the laboratory often require waveform generators capable of recreating the \\ various disturbances present in an electrical system. These sources of waveforms are generally based on \\ arbitrary waveform generators (AWG) because of the great flexibility they offer. However, AWGs are \\ generally not well suited for time varying waveforms because of the limited size of their memory. To \\ circumvent this limitation, a real time waveform synthesizer is required, but their applicability is generally \\ limited by cost. This paper presents the use of CSound, a software sound synthesizer to achieve this aim \\ in a cost effective manner. This paper first demonstrates the suitability of CSound for this application and \\ then describes how the various PQ disturbances are implemented. The design of a graphical user \\ interface which permits flexible and optimal use of the waveform generator is also presented.

\section{Keywords} \\ arbitrary waveform generators, CSound, direct synthesis, electronic music, power quality, power quality \\ disturbance, user interface

\section{Disciplines} \\ Physical Sciences and Mathematics

\section{Publication Details} \\ This article was originally published as: Braun JP, Gosbell VJ \& Perera S, Power quality waveform \\ generator based on the CSound software sound synthesizer, 11th International Conference on Harmonics \\ and Quality of Power, 12-15 September 2004, 391-396. Copyright IEEE 2004.
}




\title{
Power Quality Waveform Generator based on the CSound Software Sound Synthesizer
}

\author{
J. P. Braun, Member, IEEE, V. J. Gosbell, Member, IEEE and S. Perera, Member, IEEE
}

\begin{abstract}
Power quality investigations in the laboratory often require waveform generators capable of recreating the various disturbances present in an electrical system. These sources of waveforms are generally based on Arbitrary Waveform Generators (AWG) because of the great flexibility they offer. However, AWGs are generally not well suited for time varying waveforms because of the limited size of their memory. To circumvent this limitation, a real time waveform synthesizer is required, but their applicability is generally limited by cost. This paper presents the use of CSound, a software sound synthesizer, to achieve this aim in a cost effective manner. This paper first demonstrates the suitability of CSound for this application and then deseribes how the various $P Q$ disturbances are implemented. The design of a Graphical User Interface which permits flexible and optimal use of the waveform generator is also presented.
\end{abstract}

Index Terms-Arbitrary waveform generator, Csound, direct synthesis, electronic music, power quality, power quality disturbance, user interface.

\section{INTRODUCTION}

$\mathrm{T}$ $\mathrm{HE}$ need to recreate power quality (PQ) disturbances in laboratory environments is essential for power quality investigations and testing. Typical PQ testing undertaken in a laboratory include the measurement of susceptibility of various electrical devices, the calibration and the verification of compliance of power analyzers to meet standards such as IEC 61000-4-30 [1]. The last two activities are becoming increasingly important as the specification of PQ levels is now used in commercial transactions, and as with any other measurable quantity, the measurement method must be well defined and verifiable metrologically.

At the core of most PQ laboratory instruments generating waveforms, one can generally find arbitrary waveform generators (AWG) as they offer much improved performance over traditional waveform generators. Their key merits are high resolution, high speed and the variety of techniques available to create the desired waveforms. However, AWGs have a finite memory size, typically ranging between a few Kbytes and several hundred Mbytes. The combination of memory size and stepping clock rate set the maximum

This work was supported by GridSense, Integral Energy and by the Australian Research Council under an ARC SPIRT grant.

The authors are with the School of Electrical, Computer and Telecommunication Engineering at the University of Wollongong, Wollongong, Australia. waveform duration as it requires some time to reload the AWG memory with another waveform. This limits the type of waveforms that can be created with an AWG. It will be shown in the next section that some $P Q$ waveforms can have requirements that exceed the capability of ordinary AWGs.

The creation of waveforms in real time with a synthesizer is a possible alternative to an AWG. As this technique is used in electronic music and most PQ disturbances are in the audio spectrum, this paper proposes the use of CSound, one of the most widely used sound software synthesizers, for the generation of PQ waveforms. In addition to its flexibility which is well beyond the requirements of this application, CSound is freely available on the web [2],[3].

Firstly, the requirements of $P Q$ waveforms are reviewed and a description of how they are created with AWGs is provided. The paper will then show how CSound can be used to implement a PQ generator aimed at testing power quality analyzers during their design and verification stages. This is followed by a short introduction to electronic music and a description of the way in which various PQ disturbances are created with CSound. Finally the design of a graphical user interface (GUN) which shields the difficulties of CSound from the user of the generator is presented.

\section{POWER QuALITY WAVEForMS}

The ideal sinusoidal power system waveform at 50 or $60 \mathrm{~Hz}$ rarely exists in practice. Parameters such as frequency, magnitude and phase permanently fluctuate as a result of the practical behavior of a power system. Further deviations are introduced by the PQ disturbances classified as continuous. These include harmonics, inter-harmonics, flicker, voltage variation and unbalance. For example, the ever-increasing presence of non-linear loads introduces harmonics that themselves fluctuate in magnitude and phase [4], [5].

Network and load switching, system faults and lightning in a power system also cause deviations from the ideal sine wave. This class of disturbances, known as discrete disturbances, includes voltage swells, sags and transients (impulsive or oscillatory). These events are only present for a very short period of time and there are long time intervals between events. Their frequency content is also much higher and can easily reach several $\mathrm{MHz}$. Furthermore, the actual shape of these discrete disturbances can vary greatly and is dependant on the cause of the event. Sags, for example, can differ greatly in depth, duration and phase jump as well as in envelope [6]-[8]. 
From the perspective of a signal, the recreation of these continuous and discrete disturbances sets some specific requirements. The first is the multiphase aspect of $P Q$ waveforms and disturbance computation. Unbalance, sag duration computation as per IEC $61000-4-30$ requires three simultaneous voltage signals. Also, the computation of power (real or reactive) in a three-phase system requires a total of six signals. But, modern power analyzers are typically capable of monitoring up to four voltages as well as four currents, the same number of signals must be available for their testing.

Other important parameters are the bandwidth and accuracy. IEC 61000-1-4 [9] classifies harmonic and interharmonics as conducted emission up to $9 \mathrm{kHz}$. The possible existence of a DC component in the power system sets the lower end of the spectrum. However, the proper recreation of lightning transients may require a bandwidth extension of up to several $\mathrm{MHz}$. The presence of voltage flicker made up of voltage fluctuations in the range of 0.05 to $35 \mathrm{~Hz}$ can drastically increase the periodicity of the basic waveform. For example, a fluctuation of $0.05 \mathrm{~Hz}$ brings the period to 20 seconds while $\pi \mathrm{Hz}$ strictly brings it to infinite. In regard to accuracy, all waveforms must be synchronized within a few microseconds and have a resolution of at least 16 bits in order to be useful for power quality analyzers [10].

Another requirement is the need to create $P Q$ scenarios made of waveforms over extended periods during which any parameter of the signal can be changed at any time in a deterministic fashion. In effect, $P Q$ disturbances measured over a given period, typically a week, are often post processed into power quality indices which summarize long data trends. For example in the European standard EN50160 [11], continuous disturbances measured over a period of at least one week are converted into a single number based on a statistical process. Another example requiring long waveforms is flicker as per IEC $61000-4-15$ [12]. The low frequencies involved and the manner in which flicker severity $\left(\mathrm{P}_{\mathrm{st}}\right.$ or $\left.\mathrm{P}_{\mathrm{ft}}\right)$ indices are defined involve a slower statistical process.

\section{POWER Quality WAVEFormS WITH AWG}

To better understand the limitations of AWG for power quality applications, a short review of published examples of such generators in PQ testing is presented. The chosen examples range from simple to complex. In the simplest case, a single cycle of the waveform is stored in the memory and replayed repetitively to make the waveform periodical. This method is known as direct synthesis and is typically used for the creation of time invariant harmonics in calibration systems [13],[14]. The lowest and highest possible frequency are respectively equal to the sampling rate divided by the memory size and half the sampling frequency while the resolution is equal to the lowest frequency.

In the case of practical PQ disturbances, the lowest required frequency is $0.05 \mathrm{~Hz}$ while the highest should be at least 9 $\mathrm{kHz}$. The first represents the lower limit of flicker while the second is the IEC limit previously mentioned. These limits set the following minimum requirements: sampling rate greater than $18 \mathrm{kHz}$ and a memory depth of at least 360,000 samples. If high bandwidth transients are to be included, both sampling rate and memory size must be increased thus increasing costs. To circumvent this limitation, the usage of two AWGs is common when flicker is to be simulated. The first generator is used to create the fundamental while the second is used to amplitude modulate the first [15]. This may prove prohibitive in a three-phase system.

A similar solution may be required if more parameters of the waveform are allowed to vary independently. For example, the simultaneous presence of flicker and phase fluctuation of a given harmonic exacerbates the constraint for periodicity in the overall waveform and may lead to the need for another independent AWG for the phase variation.

Another limitation of basic AWGs is their inability to recreate discrete disturbances in a non-periodical fashion. To circumvent this problem more sophisticated AWGs with features such as external trigger input and segmented memory burst mode are required [16]. With these enhancements it is possible to store multiple waveform segments and replay them in a programmed sequence or at the occurrence of a trigger. This allows the creation of both continuous and discrete disturbances, but a computer is required to control the AWG operation, typically through a GPIB interface thus leading to an increase in system complexity and cost.

The increased capabilities described above mean that the user needs to be able to control the editing of the desired $P Q$ waveforms with little effort. An example of such a system based on LabVIEW permits the creation of complex PQ waveforms through the interconnection of functional blocks [17]. Another example of "waveform editing" is based on the assembly of waveform segments containing specific disturbances [18].

The simultaneous fluctuation of frequency, magnitude and phase on fundamental harmonics and interharmonics still remains difficult to achieve even for more sophisticated AWGs. The various rates of change may lead to extremely long periods that may be too long to be stored in memory, especially if the rates of variation change over time. To recreate such waveforms with multiple fluctuations, special types of AWGs have been developed [19]. However, their cost is very high.

Further improvements in the realization of $P Q$ waveforms have been achieved through the design of real time simulators where the device under test is "in the loop" [20]. In this system, a computer calculates every single point according to a model in real time (eg RTDS using PSCAD/EMTDC) while simultaneously accepting and sending signals to the device under test. Although such systems perform extremely well cost is a prohibitive factor.

\section{POWER QUALITY WAVEFORMS WITH CSOUND}

The desire to extend the range of available orchestral instruments has led musicians to the creation of sounds through synthesis [21]. The origin of electronic music can be traced to the end of World War II and was initially limited to 
the creation and processing of waveform with analog circuits such as oscillators, filters and non-linear circuits. The various modules were interconnected through a patch panel that allowed flexible reconfiguration. Modern day computers offer new possibilities as their computational power allows for more complex and rapidly changing waveforms to be recreated.

The first musical software, Music 1, was created in 1957 at Bell Laboratories. It has many descendants that operated on the prevailing platforms at the time of their creation. The advent of $\mathrm{PCs}$ and the $\mathrm{C}$ programming language culminated in the development of CSound at MIT in 1985. The portability of the $\mathrm{C}$ code, together with the release of CSound source code to the public domain means that CSound has become the most widely used software for sound synthesis systems. It can operate on various platforms and operating systems. Due to the high performance of today PCs, CSound is now capable of performing in real time over several channels.

A musical synthesizer must create a signal representing the sound pressure wave whose sampling rate is typically 44.1 $\mathrm{kHz}$ in audio applications. This creates two fundamental problems [22]: the first is the large amount of data required to specify a given pressure wave while the second is the need for a simple language to describe the complex sound waveform. In effect, in the case of a real time waveform, the computing time of a waveform must be less than the actual waveform duration. To reduce the computational requirements, CSound exploits the fact that many sounds are periodical and repetitive thus enabling the use of lookup tables. Interconnected block diagrams called opcodes are used to describe the sound. They are communicated to CSound via two text files: the orchestra (orc) and the score (sco). As the names indicate, the orchestra file is the collection of the various instruments built with the opcodes while the score file contains the notes.

Figure 1 provides an example of a simple sinusoidal oscillator. The symbol on the right is an OSCIL opcode that represents a simple table-lookup oscillator. The symbols on the left side show the parameters contained in the score file. In addition to waveform tables, CSound also permits mathematical computations and combination of signals as well as signal processing. At this point it should be noted that the strength of CSound lies in its ability to create a large number of small AWGs in the form of look up tables and interconnect these to process the signals. In other words CSound possesses some of the key features of a more advanced AWG as described in the previous section. It thus constitutes a good trade off between cost and performances. Figure 2 shows all the opcodes used to create $\mathrm{PQ}$ waveforms. At the top of each block are the parameters that are provided by the score file.

LINSEG is an envelope generator made up of linear segments between points defined by the users.

OSCILI is an oscillator whose waveform shape is contained in a look up table. Its interpolating capability differentiates it from OSCIL. Unlike an AWG where each sample of the output stream corresponds to a sample in memory, a sample in CSound can actually fall between two points in the table.

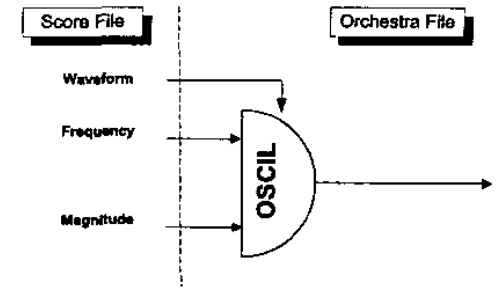

Fig. 1. CSound OpCodes used to periodical wave
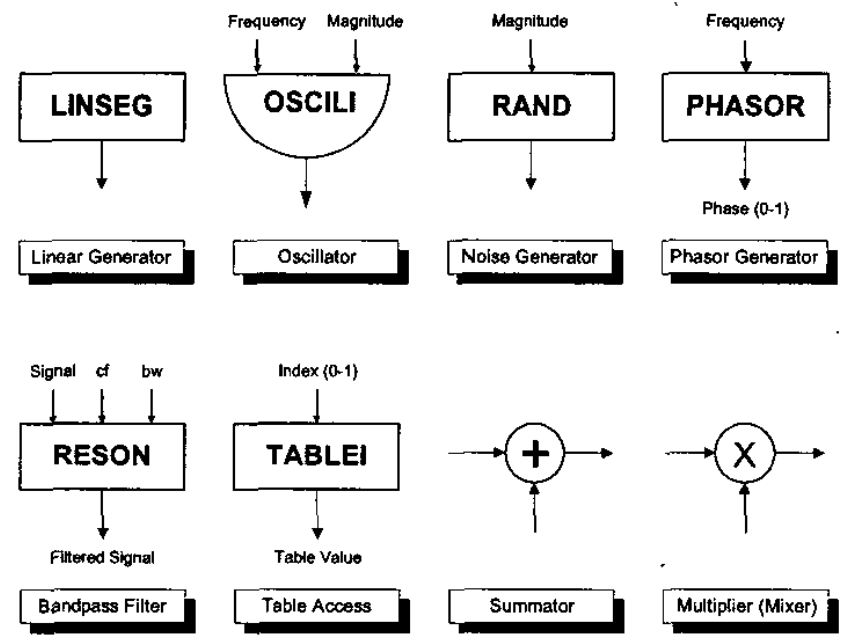

Fig. 2. CSound OpCodes used to generate $P Q$ waveforms

RAND is a pseudo random generator that can be used as a white noise generator.

PHASOR is a phasor generator that outputs a number between 0 and 1 representing a normalized instantaneous phase angle. The frequency is set in the score file.

RESON is a second order band pass filter whose center frequency as well as bandwidth can be controlled by the users.

TABLEI converts the instantaneous phase angle into the corresponding table index. This opcode permits the establishment of a direct relationship between the instantaneous phase angle and the corresponding sample or its interpolation.

SUMMATOR permits the addition of two or more signals

MULTIPLIER permits the multiplication of two signals

\section{A. CSound $P Q$ Waveform Generator}

The CSound PQ waveform generator was designed with the following objectives:

- Single phase or three phase synthesis (with neutral)

- Voltage only or voltage and current configuration

- Deterministic control of all waveform parameters

- Possibility of creating PQ scenarios.

Figure 3 shows the block diagram of a single phase $P Q$ waveform generator. The additive structure permits the addition or removal of a given disturbance to optimize performance. The GUI performs this selection automatically based on the waveform to be created. Also shown on the right of the diagram are the various fluctuation generators. 


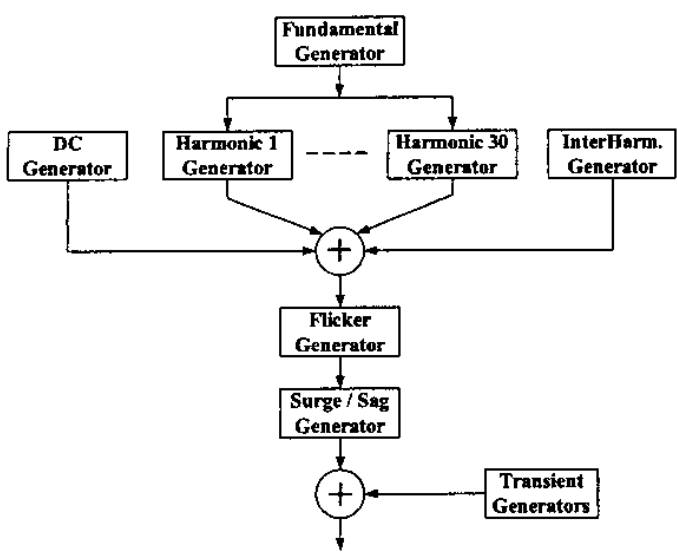

Fig. 3. Block diagram of a single phase $P Q$ waveform generator

\section{B. Power Frequency Generator and Harmonics}

In order to synchronize the phase of all waveforms, a common reference signal used by all harmonic generators must be available. Figure 4 shows the use of a single PHASOR opcode which permits this. Its input is a simple constant representing the system frequency. Frequency fluctuation can be simply caused by adding a variation signal to the constant. This signal originates from a variation generator described in the next section. The output PHASOR is a simple normalized signal representing the instantaneous phase of the power system. The instantaneous phase is fed to a summator allowing the addition of a phase step which typically occurs during a sag.

The new value of the instantaneous phase is then fed into all the harmonic generators which are made up of two summators, one multiplier and one TABLEI opcode. They convert a phase angle to a sample in a lookup table containing a single cycle of a sinusoidal waveform. The multiplication of the instantaneous phase by the harmonic rank gives the phase of a harmonic. Further addition of a constant is used in the case of a three-phase system. The final addition of a phase fluctuation from a variation generator introduces phase variation that can be static or fluctuating as shown in Figure 6.

\section{Variation Generator}

Frequency, phase and magnitude variations are created with the generator shown in Figure 5. It is capable of creating fluctuations that are sinusoidal, square, triangular or random. In the latter case, a band pass filter whose center frequency and bandwidth are under the control of the user is used.

\section{Interharmonics}

By their very nature, interharmonics do not require synchronization and each one has its own PHASOR and TABLEI opcode. The rest of the implementation is identical to harmonics. Figure 7 shows an interharmonic of $722.33 \mathrm{~Hz}$.

\section{E. Flicker}

Flicker is achieved through amplitude modulation of the signal containing harmonics and interharmoniccs. The modulation source is a variation generator. Figure 8 shows random flicker.

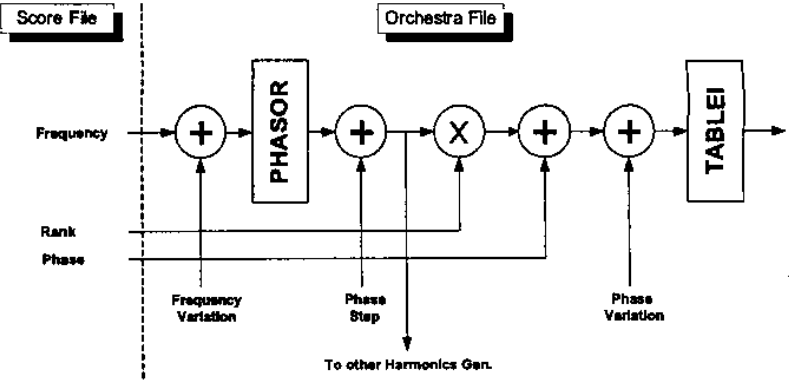

Fig. 4. Frequency generator and single harmonic generator

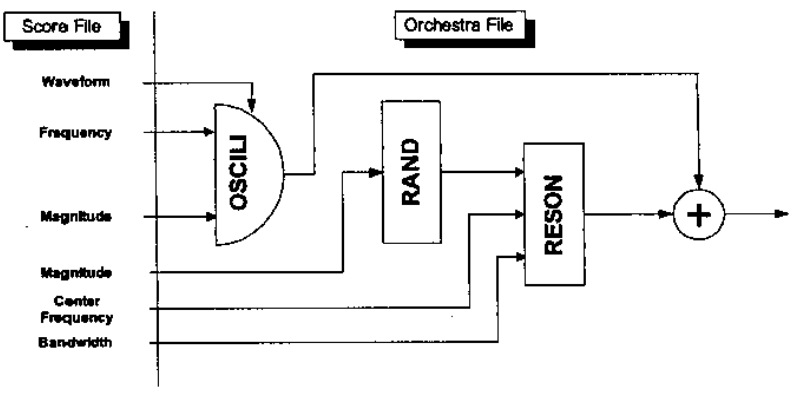

Fig 5. Block diagram of a variation generator

\section{F. Sags and Surges}

The sags and surges are simply created by multiplying a waveform by a rectangular envelope whose magnitude corresponds to the depth of the sag. The fall and rise time of the leading and trailing edge are also under the control of the user. An example is shown in Figure 9. Figure 10 shows how the usual phase jump observable in sags is reproduced with a triangular leading edge. The phase is changed in proportion to the instantaneous voltage magnitude reduction. In this case the final phase change is 50 degrees.

\section{G. Oscillatory Transients}

A sine wave generator based on the OSCILI opcode whose output magnitude is controlled by a decaying exponential makes up oscillatory transients. It is, therefore, possible to control the frequency as well as the damping time constant. The total duration of the transient is set to 12 time constants to ensure that its last sample is below the 16 bits quantification level. This resolution also sets the limits of the peak value of the transient with respect to the fundamental. Figure 11 shows a transient with an initial value of $-250 \mathrm{~V}$ and whose frequency is $670 \mathrm{~Hz}$ with a decay time constant of $4 \mathrm{mS}$

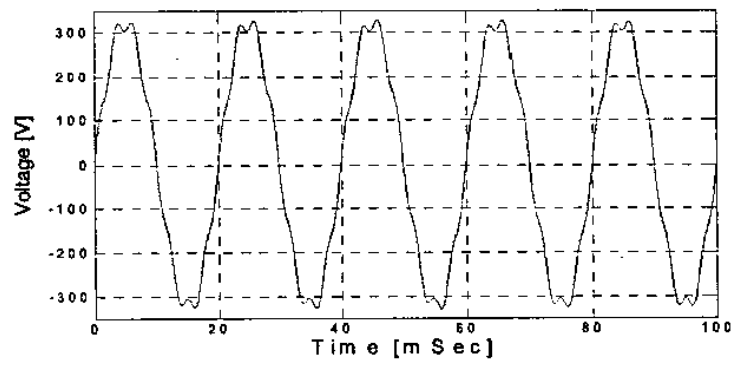

Fig. 6. Fundamental with phase fluctuating seventh harmonic 


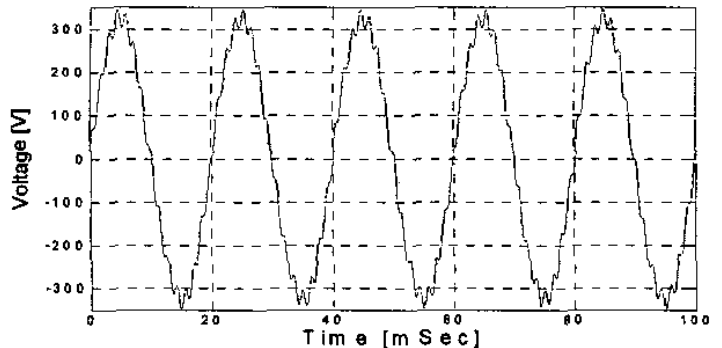

Fig. 7. Fundamental with a $722.33 \mathrm{~Hz}$ interharmonic

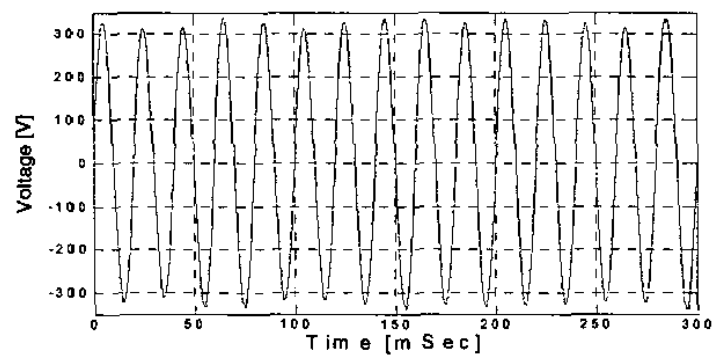

Fig. 8. Flicker with random envelope

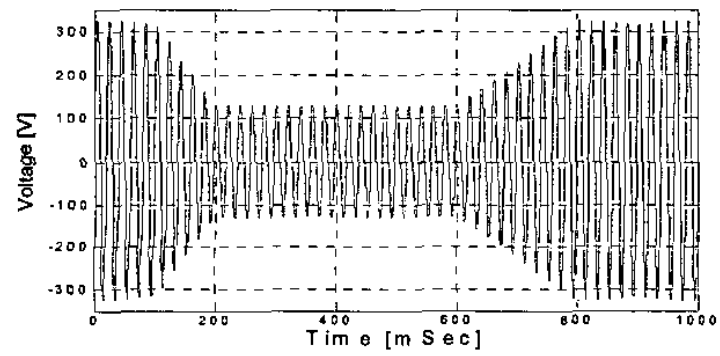

Fig. 9. Sag with a $60 \%$ depth and triangular leading and trailing edges

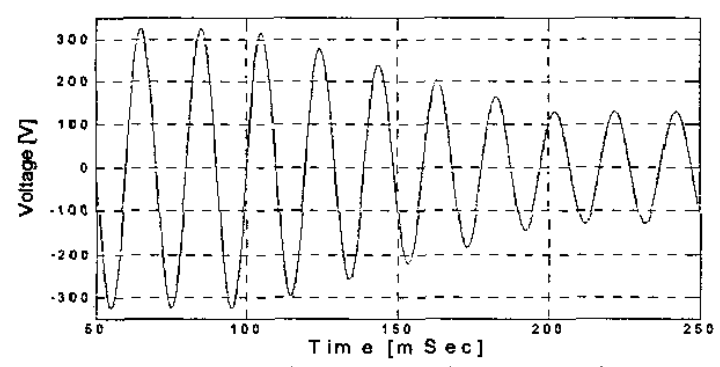

Fig. 10. Detail of the linear phase change at the beginning of sag

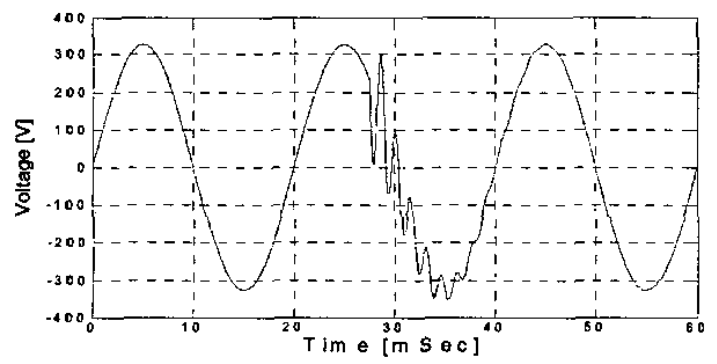

Fig. 11. Oscillatory transient associated with the switching of a PF capacitor

\section{GRAPHICAL USER INTERFACE}

With up to eight possible power system measurement configurations combined with nearly endless possibilities of PQ scenarios, manual editing of orchestra and score files is cumbersome. Additionally, the permanent inclusion of all disturbance generators is likely to exceed the capacity of a PC for real time waves. In effect, a single harmonic whose magnitude and phase fluctuate requires three oscillators. Eight signals each containing 50 harmonics would require a total of 1200 independent oscillators. While the limits of CSound for $P Q$ applications are still being investigated, it is reasonable to assume that practical scenarios will be less demanding.

The task of creating and optimizing the usage of CSound is delegated to a GUI. In addition to facilitating the creation of power quality waveforms, the GUI shields the intricacies of CSound from the user. Figure 12 shows the main window of the interface which enables the user to set the system configuration and parameters, edit PQ scenarios and start the waveform generation itself. The drop down menu in the main window permits the control of the various disturbances.

Figure 13 shows the power frequency window with two entries. These allow deviation from the nominal $50 \mathrm{~Hz}$ by $0.5 \mathrm{~Hz}$, first linearly for 20 seconds and then sinusoidally for 30 seconds. Figure 14 shows how the $3^{\text {rd }}$ and $5^{\text {th }}$ harmonics magnitudes as well as their magnitude fluctuations are set. The control of oscillatory transients is shown in Figure 15. Waveforms can either be stored on the computer's hard disk or sent to the application via a sound card and amplifier.

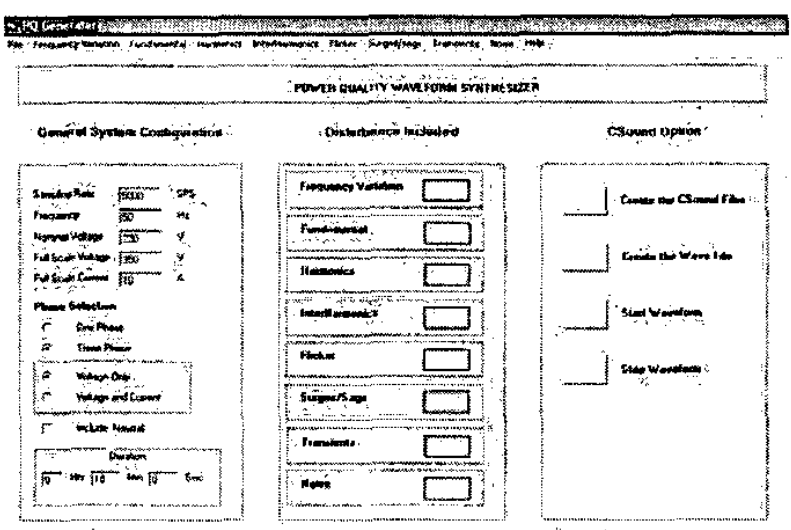

Fig. 12. Main window of the Graphical User Interface

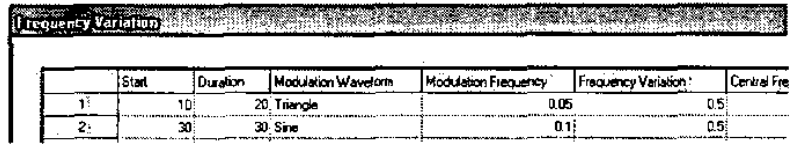

Fig. 13. Power frequency variations window

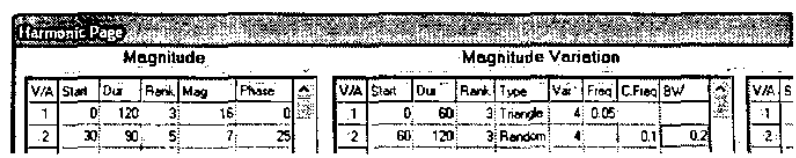

Fig. 14. Harmonics window 


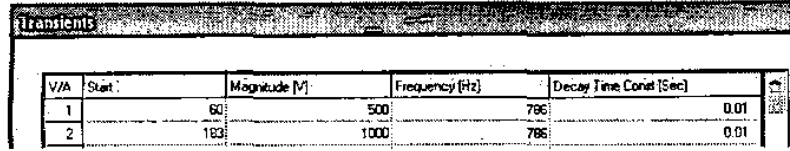

Fig. 15. Oscillatory transients window.

\section{CONCLUSIONS}

A power quality waveform based on the CSound software sound synthesizer has been presented. This generator is capable of creating up to eight independent waveforms containing several continuous or discrete disturbances simultaneously. In addition, all parameters of disturbance can be made time variant in either a deterministic or random fashion. The versatility of this generator makes it well suited for the verification or evaluation of a power quality analyzer and as a general-purpose PQ source for PQ testing.

The usage of CSound permits the achievement of performance that would normally require sophisticated and costly arbitrary waveform generators. While the abilities and performance characteristics of CSound are still being investigated at the time of writing, this synthesizer has been demonstrated to be capable of creating complex time varying waveforms at a low cost. The use of a graphical user interface further enhances the capability of this generator by allowing the user to construct the PQ scenarios for the testing analyzers.

\section{REFERENCES}

[1] Standard IEC 61000-4-30, Electromagnetic compatibility: Testing and measurement techniques: Power quality measurement methods, Feb. 2003.

[2] R. Boulanger, The CSound book. Perspectives in software synthesis, sound design, signal processing, and programming, Cambridge: MIT Press, 2000.

[3] CSound software. Available: http://www.csounds.com

[4] A. Mansoor, W. M. Grady, A. H. Chowdhury, M. J. Samotyj, "An investigation of harmonics attenuation and diversity among distributed single-phase power electronic loads," IEEE Trans. Power Delivery, vol. 10, pp. 467-473, Jan. 1995.

[5] A. Mansoor, W. M. Grady, R. S. Thallam, M. T. Doyle, S. D. Krein, M. J. Sanotyj, "Effect of supply voltage harmonics on the input current of single-phase diode bridge rectifier loads," IEEE Trans. Power Delivery, vol. 10, pp. 1416-1422, Jul. 1995.

[6] M. H. Bollen, Understanding power quality problems: Voltage sags and interruptions, New-York: IEEE Press, 2000, p.139.

[7] E. Styvaktakis, M. H. J. Bollen, I. Y. H. Gu, "Classification of power system events: Voltage dips," in Proc. $9^{\text {th }}$ Int. IEEE Conf. Harmonics and Quality of Power, 2000, vol. 2, pp. 475-750.

[8] E. Styvaktakis, M. H. J. Bollen, "Signature of voltage dips: transformer sahuration and multistage dips," IEEE Trans. Power Delivery, vol. 18, pp. 265-270, Jan. 2003.

[9] First draft Standard IEC 61000-1-4, EMC: Rationale for limiting power-frequency conducted harmonic and interharmonic current emissions from equipment, in the frequency range up to $9 \mathrm{kHz}$, Feb. 2001.

[10] S. Chen, "A quantitative analysis of the data acquisition requirements for measuring power quality phenomena," IEEE Trans. Power Delivery, vol. 18 , pp. 1575-1577, Oct. 2003.

[11] Standard EN 50160, Voltage characteristics of electricity supplied by public distribution systems, 2000.

[12] Standard IEC 61000-4-15, Electromagnetic compatibility: Testing and measurement rechniques: Flickermeter - Functional and design specifications, Feb. 2003.
[13] R. Arseneau, P. Filipski, "A calibration system for evaluating the performance of harmonic power analyzers," IEEE Trans. Power Delivery, vol. 10, pp. 1177-1182, Jul. 1995.

[14] R. Arseneau, "Calibration system for power quality instrumentation, " IEEE Power Engineering Society Winter Meeting, 2002, vol. 2, pp. 1437-1440.

[15] R. Arseneau, M. E. Sutherland, J. J. Zelle, "A test system for calibrating flickermeters," IEEE Trans. Instrumentation and Measurement, vol. 51, pp. 598-600, Aug. 2002.

[16] User Manual: PXA 125, $125 \mathrm{MS} / \mathrm{s}$ Atbitrary Waveform Generator, Publication No. 010520, LeCroy Corp., Chestnut Ridge, N.Y., Available: http://www.lecroy.com

[17] E. Matheson, A. von Jouanne, A. Wallace, "A remotely operated power quality test platform based on a $120 \mathrm{kVA}$ fully programmable thee-phase source," Conference Record of the IEEE Industry Applications Conference, $36^{\text {th }}$ IAS Annual Meeting, 2001, vol. 2, pp. 1188-1195.

[18] M. Kezunovic, Y. Liao, "A novel method for equipment sensitivity study during power quality events," IEEE Power Engineering Society Winter Meefing, 2000, vol. 2, pp. 993-998.

[19] Technical Manual: Model V370 4-Channel VME Waveform Generator, Highland Technology, Inc., San-Francisco, CA., Available: http:/www.highlandtechnology.com

[20] E. Acha, O. Anaya-Lara, J. Parle, M. Madrigal, "Real-time simulator for power quality disturbance applications", in Proc. $9^{\text {th }}$ Int. IEEE Conf. Harmonics and Quality of Power, 2000, vol. 3, pp. 763-768.

[21] A. Douglas, Electronic music production, London: Pitman, 1969, p. 5.

[22] M. V. Mathews, The technology of computer music, Cambridge: MIT Press, 1969, p. 34

\section{BIOGRAPHIES}

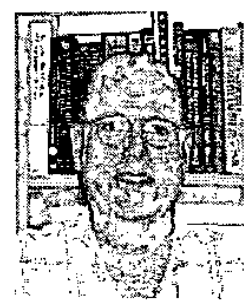

John Braun (M'1992) John Braun obtained his BE in 1980, MEM in 1993 and MEngSc in 2000 respectively from the Ecole d'Ingenieurs de Genève, Switzerland, University of Technology Sydney and University of New South Wales. He is presently a $\mathrm{PhD}$ candidate at the University of Wollongong. He has more than twenty years experience in product development and has held positions of hardware design engineer, systems engineer and group manager. His current interests lie in the field of power quality instrumentation, monitoring and signal processing.

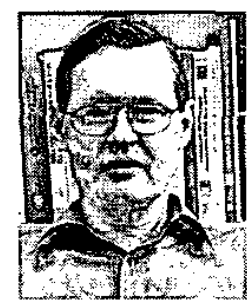

Vic Gosbell (M'1972) Dr Gosbell obtained his BE degree in 1966 and his $\mathrm{PhD}$ in 1971 from the University of Sydney. He has held academic positions at the University of Sydney and the University of Wollongong where be is foundation Professor of Power Engineering and Technical Director of the Integral Energy Power Quality Centre. He is currently working on harmonic management and power quality monitoring methodologies. He led Australia's furst national power quality survey program completed last year. $\mathrm{He}$ is a member of Australian standards and CIGRE sub-committees and is a Fellow of the Institution of Engineers, Australia.

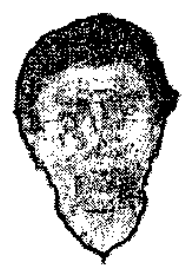

Sarath Perera (M'1995) Dr Perera graduated from the University of Moratuwa, Sri Lanka with a BSc (Eng) degree (1974) specialising in Electrical Power Engineering. He obtained his MEngSc degree (1978) from the University of New South Wales and $\mathrm{PhD}$ degree (1988) from the University of Wollongong. He is now a Senior Lecturer at the University of Wollongong. His research interests are in the area of Power Quality. 